ИЗВЕСТИЯ АКАДЕМИИ НАУК ЭСТОНСКОП ССР, ТОМ 28 ФИЗИКА * МАТЕМАТИКА. 1979, № 4

\title{
МАКСИМИЗАЦИЯ ФУНКЦИИ ВЕРОЯТНОСТИ НА ПРОСТЫХ МНОЖЕСТВАХ
}

\author{
(Представлена Х. Абеном)
}

1. Рассмотрим задачу стохастического программирования в $r$-мерном евклидовом пространстве $R^{r}$

$$
\max _{x \in X} P_{\xi}[f(x, \xi)<t] .
$$

Здесь $\xi=\xi(\omega)$ - вектор случайных параметров, отображающнй вероятностное пространство $(\Omega, \Sigma, P)$ в $\left(R^{m}, B, P_{\xi}\right) ; f(x, \xi)$ - действительная функция, определенная на $R^{r} \times R^{m} ; t$ - фиксированное число, $P_{\xi}(\cdot)$ - индуцированная вектором $\xi$ мера в $R^{m}$. Множество $X \subset R^{r}$ «простое», т. е. на $X$ можно эффективно решать вспомогательные задачи типа максимизации линейной или квадратичной функции $\left[{ }^{1}\right]$. В дальнейшем $X$ будем считать выпуклым компактом.

Решение задачи (1), с помощью итеративных методов, нспользующих значения функции $v(x, t)$ и ее производной, возможно лишь в редких случаях даже при известном распределении случайного вектора $\xi$ в выражении

$$
v(x, t)=P_{\xi}[f(x, \xi)<t],
$$

что связано с большими вычислительными трудностями из-за размерности пространства $R^{m}$. Во многих же задачах законы распределения вовсе не заданы. Поэтому значительный интерес представляют прямые методы решения задачи (1), основанные на оценках функции вероятности $v(x, t)$ и ее производной. В $\left[{ }^{2}\right]$ был рассмотрен метод максимизации функции $v(x, t)$, который заключался в замене производной от $v(x, t)$ по $x$ оценкой ее конечно-разностной аппроксимации. В основу такого подхода был положен построенный в $\left[{ }^{3}\right]$ алгоритм минимизации функции псевдоградиентным методом. Однако заданные в $\left.{ }^{2}\right]$ условия, при которых $v_{x}^{\prime}(x, t)$ удовлетворяет условию Липшица, весьма трудно поддаются проверке. Поэтому более удобной оказывается методика, разработанная Е. А. Нурминским [ $\left.{ }^{4}\right]$, которая позволяет обобщить теоремы Зангвилла $\left[{ }^{5}\right]$ о сходимости алгоритмов решения задач нелинейного программирования.

Пусть $X^{*}$ - множество решений задачи (1). Выбор метода для ее решения зависит от описания множества $X^{*}$. Если $v(x, t)$ дифференцируема по $x$, то множество решений $X^{*}$ можно представить в виде

$$
X^{*}=\left\{x^{*} \in X:\left(v^{\prime}{ }_{x}\left(x^{*}, t\right), x-x^{*}\right) \leqslant 0 \text { для всех } x \in X\right\}
$$




$$
X^{*}=\left\{x^{*} \in X: x^{*}=\pi\left(x^{*}+\varrho v^{\prime}{ }_{x}\left(x^{*}, t\right)\right) \text { для всех } \varrho>0\right\},
$$

где $\pi(y)$ - проекция точки $y$ на множество $X$. Метод линеаризации и метод проекции градиента можно рассматривать как итерационные способы проверки условий (3) и (4) соответственно. Метод для решения задачи (1) считается сходящимся, если предел любой почти наверное (п. н.) сходящейся подпоследовательности $x_{n_{k}}$ последовательности $x_{n}$, построенной каким-либо методом, принадлежит п. н. множеству $X^{*}$.

При каждом фиксированном $x$ функция $v(x, t)$ от $t$ является функцией распределения случайной величины $f(x, \xi)$. Пусть $\xi_{1}, \ldots, \xi_{n}-$ независимые случайные векторы, распределение которых совпадает с распределением вектора $\xi$. Тогда $f\left(x, \xi_{1}\right), \ldots, f\left(x, \xi_{n}\right)-$ независимые случайные величины с распределением $v(x, t)$. Для построения дифференцируемой по $x$ оценки функции $v(x, t)$ воспользуемся оценкой Парзена $\left[{ }^{6}\right]$, которая в данном случае принимает вид

$$
v_{n}\left(x, t, \xi_{1}, \ldots, \xi_{n}\right)=\frac{1}{n h_{n}} \sum_{i=1}^{n} \int_{-\infty}^{t} K\left(\frac{\tau-f\left(x, \xi_{i}\right)}{h_{n}}\right) d \tau,
$$

где $\lim _{n \rightarrow \infty} h_{n}=0, \quad \lim _{n \rightarrow \infty} n h_{n}=\infty$,

и непрерывная функция действительной переменной $K(y)$ удовлетворяет условиям

$$
\begin{array}{ll}
\int_{-\infty}^{\infty} K(y) d y=1, & \sup _{-\infty<y<\infty}|K(y)|<\infty, \\
\int_{-\infty}^{\infty} y K(y) d y=0, & \int_{-\infty}^{\infty}|y K(y)| d y<\infty .
\end{array}
$$

Производную оценки $v_{n}\left(x, t, \xi_{1}, \ldots, \xi_{n}\right)$ можно выписать в следующем виде

$$
v_{n x}^{\prime}\left(x, t, \xi_{1}, \ldots, \xi_{n}\right)=-\frac{1}{n h_{n}} \sum_{i=1}^{n} f_{x}^{\prime}\left(x, \xi_{i}\right) K\left(\frac{t-f\left(x, \xi_{i}\right)}{h_{n}}\right) .
$$

Условия дифференцируемости функции $v(x, t)$ по $x$ довольно громоздки и малоинформативны. Один вариант таких условнй приведен В $\left[{ }^{7}\right]$. В дальнейшем будем предполагать, что производные $v_{x}^{\prime}(x, t)$ и $v^{\prime \prime}{ }_{x t}(x, t)$ существуют для всех $x \in X$ и $t \in R^{1}$.

Л е мм а. Если $v_{x t}^{\prime \prime}(x, t)$ равномерно ограничена для всех $t \in R^{1}$, $\boldsymbol{x} \in X$ и сущзествует числовая функция $R(\xi)$ такая, что

$$
\int_{R^{m}} R(\xi) d P_{\xi}(\xi)<\infty \quad \text { u }\left\|f^{\prime}{ }_{x}(x, \xi)\right\| \leqslant R(\xi)
$$

при любом $x \in X$, тогда $v_{n x}^{\prime}\left(x, t, \xi_{1}, \ldots, \xi_{n}\right)$ сходится $n$. н. равномерно no $x \in X \kappa v_{x}^{\prime}(x, t)$.

Доказ а тельство. При сделанных предположениях о $K(y)$ и $f^{\prime}{ }_{x}(x, \xi)$ справедлив аналог следствия $(4.1)$ из $\left[{ }^{8}\right]:$

$$
P\left[\lim _{n \rightarrow \infty} \sup _{x \in X}\left\|v_{n x}^{\prime}\left(x, t, \xi_{1} \ldots, \xi_{n}\right)-E v_{n x}^{\prime}\left(x, t, \xi_{1}, \ldots, \xi_{n}\right)\right\|=0\right]=1,
$$

т. е. $v_{n x}^{\prime}\left(x, t, \xi_{1}, \ldots, \xi_{n}\right)$ сходится п. н. равномерно по $x \in X \quad$ к 
$E v_{n x}^{\prime}\left(x, t, \xi_{1}, \ldots, \xi_{n}\right)$, где $E-$ знак математического ожидания. Известно $\left[{ }^{9}\right]$, что

$$
E v_{n x}^{\prime}\left(x, t, \xi_{1}, \ldots, \xi_{n}\right)=v_{x}^{\prime}(x, t)-h_{n} \int_{-\infty}^{\infty} y K(y) v_{x t}^{\prime \prime}\left(x, t-\theta h_{n} y\right) d y,
$$

где $0 \leqslant \theta \leqslant 1$. При условиях, наложенных на равномерную ограниченность $v^{\prime \prime}{ }_{x t}(x, t)$, на $K(y)$ и на $h_{n}$, имеем, что

$$
\lim _{n \rightarrow \infty} E v_{n x}^{\prime}\left(x, t, \xi_{1}, \ldots, \xi_{n}\right)=v_{x}^{\prime}(x, t)
$$

равномерно на множестве $X$. Суммируя (9) и (10), получим требуемый результат.

2. Метод линеаризации. Определим множество решений $X^{*}$ задачи (1) в виде (3). Рассмотрим случайную последовательность $x_{n}$, заданную соотношениями

$$
\begin{gathered}
x_{0} \in X, \\
x_{n+1}=x_{n}+\mathrm{Q}_{n}\left(\bar{x}_{n}-x_{n}\right), \\
\left(v_{n x}^{\prime}\left(x_{n}, t, \xi_{1}, \ldots, \xi_{n}\right), \bar{x}_{n}\right)=\max _{x \in X}\left(v_{n x}^{\prime}\left(x_{n}, t, \xi_{1}, \ldots, \xi_{n}\right), x\right),
\end{gathered}
$$

где $v_{n x}^{\prime}\left(x_{n}, t, \xi_{1}, \ldots, \xi_{n}\right)$ дается соотношением (8).

Разъясним случайную природу последовательности $x_{n}$. При каждом $n$ случайный вектор $x_{n}$ определяется на б-алгебре $F_{n-1}$, порожденной случайными векторами $\xi_{1}, \ldots, \xi_{n-1}$. Отдельные реализации случайной последовательности $x_{n}$ как случайные величины задаются на пространстве представлений с $\sigma$-алгеброй $F$, индуцированной расширяющейся последовательностью $\sigma$-алгебр $F_{n}$. Это пространство мы обозначим через $(\Xi, F, \mu)$, где $\mu(\Xi)=1, \Xi=\prod_{i=1}^{\infty} R_{i}^{m}$ и $\mu(\cdot)-$ индуцированная мера на $(\Xi, F)$. Элементы множества $\Xi$ обозначим через $\xi^{\infty}$. Т еорем а 1. Если выполнены условия леммы, $0 \leqslant \mathrm{Q}_{n} \leqslant 1, \mathrm{Q}_{n} \rightarrow 0$, $\sum_{n=1}^{\infty} Q_{n}=\infty$ и v $(x, t)$ принимает на $X^{*}$ конечное число значений, то предельные точки последовательности $x_{n}$ принадлежат п. н. множеству $X^{*}$.

Д ок а з а тельство. Воспользуемся методикой из $\left[{ }^{4}\right]$. Пусть существует подпоследовательность $x_{n_{k}}$, которая сходится, но не к точке множества $X^{*}$, т. е.

$$
\lim _{k \rightarrow \infty} x_{n_{k}}\left(\xi^{n_{k-1}}\right)=x^{\prime}\left(\xi^{\infty}\right) \notin X^{*} \text {, где } \xi^{s}=\left(\xi_{1}, \ldots, \xi_{s}\right) \text {, }
$$

для всех $\xi^{\infty} \in B \subset \Xi$ и $\mu(B)>0$. Без ограничения общности примем, что $B=\Xi$. Тогда для любого $\varepsilon_{1}>0$ найдется $\Delta>0$ такая, что $\mu\left(\Xi_{1}\right) \geqslant 1-\varepsilon_{1}$, где

$$
\Xi_{1}=\left\{\xi^{\infty}:\left(v_{x}^{\prime}\left(x^{\prime}\left(\xi^{\infty}\right), t\right), x-x^{\prime}\left(\xi^{\infty}\right)\right) \geqslant \Delta\right\} .
$$

В дальнейшем будем считать, что $\xi^{\infty} \in \Xi_{1}$. В силу непрерывности функции,$\left(v^{\prime}{ }_{x}(x, t), y-x\right)$ по $x$ найдется индекс $N=N\left(\xi^{\infty}\right)$ такой, что при $k \geqslant N$ и всех $n \geqslant n_{k}$

$$
\left(v_{x}^{\prime}\left(x_{n_{k}}, t\right), x-x_{n}\right) \geqslant \Delta / 2
$$

для всех $x \in X$. Покажем, что вся последовательность $x_{n}$, начиная с некоторого номера $n_{k}$, не может находиться внутри $\varepsilon$-окрестности точки $x_{n_{k}}$. Допустим существование $N^{\prime}\left(\xi^{\infty}\right)$ такого, что при достаточно 
малом $\varepsilon$ и всех $k>N^{\prime}$ имеет место $\left\|x_{s}-x_{n_{k}}\right\|<\varepsilon$ для любого $s>n_{k}$. Тогда

$$
\begin{gathered}
v\left(x_{s}, t\right)-v\left(x_{n_{k}}, t\right)=\left(v^{\prime}{ }_{x}\left(x_{n_{k}}, t\right), x_{s}-x_{n_{k}}\right)+o(\varepsilon)= \\
=\left(v_{x}^{\prime}\left(x_{n_{k}}, t\right), \sum_{n=n_{k}}^{s-1} \varrho_{n}\left(\bar{x}_{n}-x_{n}\right)\right)+o(\varepsilon) .
\end{gathered}
$$

Согласно лемме $1, v_{n x}^{\prime}\left(x, t, \xi_{1}, \ldots, \xi_{n}\right)$ сходится п. н. равномерно по $x \in X$ к $v_{x}^{\prime}(x, t)$, т. е. для любого $\delta>0$ при всех $\xi^{\infty} \in \Xi_{1}$ найдется $N^{\prime \prime}{ }_{\delta}\left(\xi^{\infty}\right)$ такой, что при любом $n \geqslant N^{\prime \prime}{ }_{\delta}\left(\xi^{\infty}\right)$

$$
\left\|v_{n x}^{\prime}\left(x, t, \xi_{1}, \ldots, \xi_{n}\right)-v_{x}^{\prime}(x, t)\right\|<\delta .
$$

Следовательно, при достаточно большом $k$

$$
\begin{aligned}
& \max _{x \in X}\left(v_{n_{k} x}^{\prime}\left(x_{n_{k}}, t, \xi_{1}, \ldots, \xi_{n_{k}}\right), x-x_{n}\right)= \\
& =\left(v_{n_{k} x}^{\prime}\left(x_{n_{k}}, t, \xi_{1}, \ldots, \xi_{n_{k}}\right), \bar{x}_{n}-x_{n}\right) \geqslant \Delta / 4
\end{aligned}
$$

и

$$
\left(v_{x}^{\prime}\left(x_{n_{k}}, t\right), \bar{x}_{n}-x_{n}\right) \geqslant \Delta / 8 .
$$

Тогда из (12) вытекает

$$
v\left(x_{s}, t\right)-v\left(x_{n_{k}}, t\right) \geqslant \Delta / 8 \sum_{n=n_{k}}^{s-1} \varrho_{n}+o(\varepsilon),
$$

что при $s \rightarrow \infty$ противоречит ограниченности левой части этого неравенства. Стало быть, существует номер

$$
m_{k}\left(\xi^{\infty}\right)=\min \left\{n: n>n_{k},\left\|x_{n}-x_{n_{k}}\right\| \geqslant \varepsilon\right\}
$$

такой, что $m_{k}<\infty$.

По определению $\left\|x_{m_{k}}-x_{n_{k}}\right\| \geqslant \varepsilon$ и $\left\|x_{m_{k}-1}-x_{n_{k}}\right\|<\varepsilon$. Но поскольку

$$
\left\|x_{m_{k}}-x_{n_{k}}\right\| \leqslant\left\|x_{m_{k}}-x_{m_{k}-1}\right\|+\left\|x_{m_{k}-1}-x_{n_{k}}\right\|
$$

и в силу ограниченности множества $X$

$$
\left\|x_{m_{k}}-x_{m_{k}-1}\right\|=\mathrm{Q}_{m_{k}-1}\left\|\bar{x}_{m_{k}-1}-x_{m_{k}-1}\right\| \leqslant \mathrm{\varrho}_{m_{k}-1} L
$$

и $\varrho n \rightarrow \infty$ при $n \rightarrow \infty$, то при достаточно большом $k$

$$
\left\|x_{m_{k}}-x_{n_{k}}\right\|<2 \varepsilon \quad \text { при } \xi^{\infty} \in \Xi_{2}, \mu\left(\Xi_{2}\right) \geqslant 1-2 \varepsilon_{1} .
$$

Следовательно, неравенство (13) остается в силе и для $s=m_{k}$, т. е.

$$
v\left(x_{m_{k}}, t\right)-v\left(x_{n_{k}}, t\right) \geqslant \Delta / 8 \sum_{n=n_{q_{k}}}^{m_{k}-1} \mathrm{Q}_{n}+o(2 \varepsilon) \text {. }
$$

Но $\varepsilon<\left\|x_{m_{k}}-x_{n_{k}}\right\| \leqslant \sum_{n=n_{k}}^{m_{k}-1} \varrho_{n}\left\|\bar{x}_{n}-x_{n}\right\| \leqslant L \sum_{n=n_{k}}^{m_{k}-1} \varrho_{n}, \quad$ откуда

$$
\sum_{n=n_{k}}^{m_{k}-1} \varrho_{n}>\varepsilon / L
$$

и

$$
v\left(x_{m_{k}}, t\right) \geqslant v\left(x_{n_{k}}, t\right)+\Delta \varepsilon / 8 L+o(2 \varepsilon) .
$$


Без ограничения общности допустим, что существуют и $\lim _{k \rightarrow \infty} v\left(x_{n_{k}}, t\right)=d^{\prime}$. Тогда $d^{\prime \prime} \geqslant d^{\prime}+\Delta \varepsilon / 8 L$.

$\lim _{k \rightarrow \infty} v\left(x_{m_{k}}, t\right)=d^{\prime \prime}$

Выберем из $\left(d^{\prime}, d^{\prime \prime}\right)$ числа $a$ и $b$ такие, что для любого $x^{*} \in X^{*}$ либо $v\left(x^{*}, t\right)<a$, либо $v\left(x^{*}, t\right)>b$. Это возможно в силу предположения о конечности числа значений $v(x, t)$ на $X^{*}$. Последовательность $v\left(x_{n}, t\right)$ пересекает интервал $(a, b)$ сверху вниз $\left[{ }^{10}\right]$ бесконечное число раз. Выберем последовательности индексов $\left\{p_{k}\right\}$ и $\left\{q_{k}\right\}$ так, чтобы $p_{k}<q_{k}$, $v\left(x_{p_{k}}, t\right) \leqslant b, v\left(x_{q_{k}}, t\right) \leqslant a, \quad a<v\left(x_{s}, t\right)<b, \quad p_{k}<s<q_{k}$. Соответствующим выбором последовательности пар $\left(p_{k}, q_{k}\right)$ всегда можно добиться того, чтобы последовательность $x_{p_{k}}$ сходилась. Пусть $\lim _{k \rightarrow \infty} x_{p_{k}}=$ $=x^{\prime \prime}$. Так как

$$
0 \geqslant b-v\left(x_{p_{k}}, t\right) \geqslant v\left(x_{p_{k}+1}, t\right)-v\left(x_{p_{k}}, t\right)
$$

и

$$
\lim _{n \rightarrow \infty}\left\|x_{n+1}-x_{n}\right\|=0 \text {, то } \lim _{k \rightarrow \infty} v\left(x_{p_{k}}, t\right)=b,
$$

следовательно, вновь можно найти номер $s_{k}<\infty$ такой, что

$$
s_{k}=\min \left\{s: s>p_{k},\left\|x_{s}-x_{p_{k}}\right\|>\varepsilon\right\} \text {. }
$$

При достаточно малом $\varepsilon>0$ и достаточно большом $k$ верно $p_{k}<s_{k}<q_{k}$. Тем самым для всех $\xi^{\infty} \in \Xi_{2}, \mu\left(\Xi_{2}\right) \geqslant 1-2 \varepsilon_{1}$

$$
{\underset{l i m}{k \rightarrow \infty}} v\left(x_{s_{k}}, t\right) \leqslant \lim _{k \rightarrow \infty} v\left(x_{p_{k}}, t\right)=b .
$$

Но неравенство (14) дает, в силу произвольности $\varepsilon_{1}>0$,

$$
\varliminf_{k \rightarrow \infty}^{\lim v} v\left(x_{s_{k}}, t\right)>\lim _{k \rightarrow \infty} v\left(x_{p_{k}}, t\right) .
$$

Полученное противоречие доказывает теорему.

3 а м е ание 1. Метод стохастической линеаризации был изучен в $\left[{ }^{11}\right]$ для локально липшицевых функций и в : $\left.{ }^{12}\right]$ для выпуклых функций.

3. Метод проекци и градиента. Определим множество решений $X^{*}$ задачи (1) в виде (4). Рассмотрим случайную последовательность $x_{n}$, заданную соотношениями

$$
\begin{gathered}
x_{0} \in X, \\
x_{n+1}=\pi\left(x_{n}+\mathrm{Q}_{n} v^{\prime}{ }_{n x}\left(x_{n}, t, \xi_{1}, \ldots, \xi_{n}\right)\right) .
\end{gathered}
$$

Т еорем а 2. Если выполнены условия теоремы 1, то предельные точки последовательности $x_{n}$, определенной соотношением (15), $n$. н. принадлежат множеству $X^{*}$, определенному соотношением (4).

Доказ а тельство. Пусть существует подпоследовательность $x_{n_{n}}$, которая сходится, но не к точке множества $X^{*}$ для почти всех $\xi^{\infty} \in \Xi$. Тогда для любого $\varepsilon_{1}>0$ найдется $\Delta>0$ такая, что $\mu\left(\Xi_{1}\right) \geqslant$ $\geqslant 1-\varepsilon_{1}$, где

$$
\Xi_{1}=\left\{\xi^{\infty}:\left\|x^{\prime}\left(\xi^{\infty}\right)-\pi\left(x^{\prime}\left(\xi^{\infty}\right)+v_{x}^{\prime}\left(x^{\prime}\left(\xi^{\infty}\right), t\right)\right)\right\| \geqslant \Delta>0\right\} .
$$

Покажем, что при достаточно большом $k$ и всех $n \geqslant n_{k}$

$$
\left(v_{x}^{\prime}\left(x_{n}, t\right), \pi\left(x_{n}+\mathrm{Q}_{n} v_{x}^{\prime}\left(x_{n}, t\right)\right)-x_{n}\right) \geqslant \mathrm{Q}_{n} \Delta^{2} / 4 .
$$


Известно, что при $0 \leqslant \varrho \leqslant 1$

и

$$
\mathrm{Q}\left\|x-\pi\left(x+v_{x}^{\prime}(x, t)\right)\right\| \leqslant\left\|x-\pi\left(x+\mathrm{\varrho}^{\prime}{ }_{x}(x, t)\right)\right\|-
$$

$$
\varrho\left(v_{x}^{\prime}(x, t), \pi\left(x+\varrho v_{x}^{\prime}(x, t)\right)-x\right) \geqslant\left\|\pi\left(x+\varrho v_{x}^{\prime}(x, t)\right)-x\right\|^{2}
$$

(см., напр., $\left.\left[{ }^{13}\right]\right)$. При достаточно большом $k$ и всех $n \geqslant n_{k}$

$$
\left\|x_{n}-\pi\left(x_{n}+v_{x}^{\prime}\left(x_{n}, t\right)\right)\right\| \geqslant \Delta / 2
$$

и

$$
\left\|x_{n}-\pi\left(x_{n}+\mathrm{Q}_{n} v_{x}^{\prime}\left(x_{n}, t\right)\right)\right\| \geqslant \mathrm{Q}_{n}\left\|x_{n}-\pi\left(x_{n}+v_{x}^{\prime}\left(x_{n}, t\right)\right)\right\| \geqslant \varrho_{n} \Delta / 2 .
$$

Из неравенства (16) получим теперь

Так как

$$
\left(v_{x}^{\prime}\left(x_{n}, t\right), \pi\left(x_{n}+\varrho_{n} v_{x}^{\prime}\left(x_{n}, t\right)\right)-x_{n}\right) \geqslant \varrho_{n} \Delta^{2} / 4 .
$$

$$
\begin{gathered}
\mid\left(v_{x}^{\prime}\left(x_{n}, t\right), \pi\left(x_{n}+\varrho_{n} v_{n x}^{\prime}\left(x_{n}, t, \xi_{1}, \ldots, \xi_{n}\right)\right)-x_{n}\right)- \\
-\left(v_{x}^{\prime}\left(x_{n}, t\right), \pi\left(x_{n}+\varrho_{n} v_{x}^{\prime}\left(x_{n}, t\right)\right)-x_{n}\right) \mid \leqslant \\
\leqslant M_{\varrho_{n}}\left(\left\|v_{n x}^{\prime}\left(x_{n}, t, \xi_{1}, \ldots, \xi_{n}\right)-E v_{n x}^{\prime}\left(x_{n}, t, \xi_{1}, \ldots, \xi_{n}\right)\right\|+\right. \\
\left.\quad+\left\|E v_{n x}^{\prime}\left(x_{n}, t, \xi_{1}, \ldots, \xi_{n}\right)-v_{x}^{\prime}\left(x_{n}, t\right)\right\|\right),
\end{gathered}
$$

где $M$ - некоторая константа, то в силу леммы и того, что $\varrho_{n} \rightarrow 0$, имеем, начиная с некоторого $k$,

$$
\left(v_{x}^{\prime}\left(x_{n_{k}}, t\right), \pi\left(x_{n}+\mathrm{Q}_{n} v_{n x}^{\prime}\left(x_{n}, t, \xi_{1}, \ldots, \xi_{n}\right)\right)-x_{n}\right) \geqslant \mathrm{Q}_{n} \Delta^{2} / 16 .
$$

Следовательно, может быть получено неравенство

$$
v\left(x_{s}, t\right)-v\left(x_{n_{k}}, t\right) \geqslant \Delta^{2} / 16 \sum_{n=n_{k}}^{s-1} \mathrm{Q}_{n}+o(\varepsilon),
$$

аналогичное неравенству (13). Остальная схема доказательства та же, что и доказательства теоремы 1.

3 ам еч ани е 2. Метод стохастической аппроксимации проекции градиента был предложен в $\left[{ }^{14}\right]$. Однако функция $v(x, t)$ не удовлетворяет заданным там условиям.

\section{Л ИТ Р А Т У Р А}

1. Поляк Б. Т., В кн.: Итоги науки и техники. Математический анализ, т. 12, М., ВИНИТИ, 1974 , с. $147-197$.

2. Ю б и Э. А.-Ю., Тр. Таллинск. политехн. ин-та, № 411, 57-76 (1976).

3. Поляк Б. Т., Цы п к и Я Я. 3., Автоматика и телемеханика, № 3, 45-68 (1973).

4. Н у р м и н с ки й Е. А., Кибернетика, № 6, 79-81 (1972).

5. З ангвилл У. И., Нелинейное программирование. Единый подход, 'M., «Сов. радио», 1973.

6. P a rze n, E., Ann. Math. Statistics, 33, № 3, 1065-1076 (1962).

7. Р а й к Э., Изв. АН ЭССР, Физ, Матем., 24, № 11, 3-9 (1975).

8. Le C a m, L., Univ. I Calif. Public. Statistics, 1, № 11, 277-330 (1953).

9. Т а м м Э., Изв. АН ЭССР, Фнз. Матем., 28, № 1, 17-24 (1979).

10. Нев ё Ж., Математические основы теории вероятностей, М., «Мир», 1969.

11. Е рм ольев Ю. М., Методы стохастического программирования, М., «Наука», 1976.

12. Гу п а л А. М., Б а же н ов Л. Г., Кибернетика, № 3, 116-117 (1972).

13. К а рм анов В. Г., Математическое программирование, М., «Наука», 1975.

14. Hiriart-Urruty, J. B., Ann. Sci. l'Univ. Clermont, № 58, 110-131' (1976). 
R. LEPP

\section{TOENÄOSUSFUNKTSIOONI MAKSIMEERIMINE LIHTSATE KITSENDUSTE KORRAL}

Olesande $\max _{x \in X} P_{\xi}[f(x, \xi)<t]$ lahendamiseks on kasutatud tingliku gradiendi ja gradiendi projektsiooni meetodit, kusjuures gradiendi hindamiseks juhusliku vektori $\xi$ realisatsioonide kaudu on kasutatud Parzeni tuuma tüüpi hinnangut $\left[{ }^{6}\right]$. Saadud jada piirpunktid rahuldavad ülesande lahendi tarvilikke tingimusi.

\section{R. LEPP}

\section{MAXIMIZATION OF THE PROBABILITY FUNCTION IN SIMPLE CONSTRAINTS}

This paper is concerned with two algorithms for finding a maximum of probability function $v(x, t)=P_{\xi}[f(x, \xi)<t]$ in $X$, where $X \subset R^{r}$ is a convex closed and bounded set, in which we can efficiently solve auxiliary linear or quadratic programming problems; $\xi$ is a $m$-dimensional random vector with unknown distribution and the real number $t$ is fixed. Basing on independent realizations of the random vector $\xi$, the Parzen kernel-type $\left[{ }^{6}\right]$ differentiable in $x$ estimate of $v(x, t)$ is constructed. It is shown that limit points of the sequences generated by linearization and gradient projection methods, satisfy the necessary conditions for maximality with the probability one. 